Richard J. Grunawalt / John E. King / Ronald S. McClain (eds.)

\title{
Protection of the Environment During Armed Conflict
}

International Law Studies, US Naval War College, Vol. 69

New Port, Rhode Island, o.J. (1997), 720 S.

In der seit 1901 erscheinenden "blue book"-Serie des US Naval War College ist der vorliegende 69. Band das Ergebnis einer kürzlichen internationalen Tagung, die sich das Ziel gesetzt hatte, dem Umweltschutz in militärischen Konflikten zu stärkerer Beachtung zu verhelfen. Unmittelbarer Anlaß waren die massiven absichtlichen Öleinleitungen und die rund 700 in Brand gesetzten Ölquellen im Golfkrieg, aber auch das wachsende Unbehagen über die Praxis der Gewaltanwendung rücksichtsloser "war-lords" und Terroristen, die zunehmend zu ökologischer Erpressung greifen.

Der innere Aufbau des Tagungsbandes in zehn Abschnitten und 39 Kapiteln ist verwirrend, folgt aber bei näherem Hinsehen einer soliden Dreiteilung: Am Anfang steht das Umweltrisiko der verschiedenen modernen Waffen und ihres Einsatzes aus technischer, wissenschaftlicher und umweltrelevanter Sicht. Es folgt eine Darstellung der bestehenden Vorschriften des Kriegsrechts und der Rechtslage für militärische Einsätze unterhalb der Schwelle eines internationalen Konfliktes, also in Fällen von Bürgerkriegen, Polizeiaktionen, humanitären Aktionen und dergleichen. Breiten Raum nimmt dabei die Haftung der Staaten, aber auch der Einzeltäter ein. Am Ende steht eine ausführliche Abwägung des Für und Wider neuer Regelungen einschließlich machtpolitischer Gegenmaßnahmen.

Im System des Kriegsrechts finden sich überraschend viele Vorschriften mit Bedeutung für den Umweltschutz, z.B. zum Verbot unnötiger Zerstörungen, zum Schutz bestimmter Objekte und Gebiete oder zum Verbot bestimmter Waffen und Methoden der Kriegführung. Im weiten Feld der "military operations other than war (MOOTW)", wozu jetzt aus Jugoslawien viele Erfahrungen vorliegen, kann sich der Umweltschutz bisher nur auf wenige allgemeine Prinzipien oder nationales und regionales Recht stützen. Hier besteht Bedarf für Neuregelungen.

Aus den Vorträgen und Diskussionsbeiträgen ergeben sich viele prüfenswerte Vorschläge, zumindest Objekte besonderer Umweltschutzbedürftigkeit zu regeln. Ein anderer Ansatzpunkt könnte in der Ausweitung der Menschenrechte und des Haftungsrechts liegen. Wo das Recht bisher versagt, ist es um so wichtiger, daß die eingesetzten Streitkräfte eine gewisse Umweltethik und Verantwortungsbewußtsein praktizieren, wie dies in den internen Dienstvorschriften vieler Streitkräfte - zumindest in denen der NATO - bereits durchgeführt ist. Die Einbeziehung des Umweltschutzes in militärische Planung, Ausbildung und Führung mußte zu diesem Zweck zu einer Selbstverständlichkeit für alle Militärs werden.

Ist es angelsächsischer Pragmatismus oder nüchterne militärische Erkenntnis, daß die praktischen Empfehlungen am Ende des Buches sich darauf beschränken, das vorhandene Recht energischer durchzusetzen anstelle von Neuregelungen? In der Zusammenfassung von John Norton Moore, einem sehr angesehenen Fachmann für Seerecht und Sicherheitspolitik, wird überdeutlich, daß allein wirksame äußere Abschreckung bzw. gezielte Intervention verbunden mit der realistischen Chance, die Haupttäter persönlich zur Verantwortung zu ziehen, geeignet 
sind, kriegerische Umweltdesperados von ökologischer Kriegführung abzuhalten. Insofern ist der Versuch der UN, Kriegsverbrecher und Menschenrechtsverletzer zur Verantwortung zu ziehen und dafür die internationalen Gerichte zu stärken, wohl das richtige Rezept.

Obwohl das Buch eine Fülle von Anregungen bietet, leidet es wie häufig bei Tagungsbänden an fachlich unterschiedlich gewichtigen und wortreichen Beiträgen, die offenbar ohne jegliche redaktionelle Straffung abgedruckt wurden. Das gute Stichwortverzeichnis bemüht sich, diesen Mangel auszugleichen. Bedenkt man, daß der Umweltschutzgedanke im Interesse der gesamten Menschheit bei der zunehmenden Zahl von menschenverachtenden Gewaltanwendungen dringend durchgesetzt werden muß, so ist diese Veröffentlichung ein guter Einstieg für Juristen, Militärs und Diplomaten, die den Umweltschutz ernst nehmen.

Uwe Jenisch

\section{Marc Leonhard}

Der ökologische Schaden. Eine rechtsvergleichende Untersuchung

Schriftenreihe der Gesellschaft für Rechtsvergleichung, Band 175

Nomos Verlagsgesellschaft, Baden-Baden, 1996, 423 S., DM 118,--

Mit den großen Umweltkatastrophen von Seveso (1976), Bhopal (1984), Tschernobyl (1986), Sandoz (1986) und Exxon Valdez (1989) verstärkte sich die Diskussion um die Ergänzung des ordnungsrechtlich und durch administrative Kontrolle geprägten Umweltrechts durch indirekte Verhaltenssteuerung und ökonomische Instrumente wie Abgaben, Steuervergünstigungen und Subventionen. Auch das Haftungsrecht kann als Kompensations- und Präventionsinstrument zu umweltgerechtem Verhalten anhalten. Rechtsvergleichende Arbeiten aus jüngerer Zeit zur Haftung für ökologische Schäden gibt es von Erich$\operatorname{sen}^{1}$ und Seibt ${ }^{2}$. Die nunmehr vorliegende Jenenser Dissertation von Marc Leonhard befaßt sich mit dem Ersatz ökologischer Schäden im deutschen, US-amerikanischen, französischen, italienischen, europäischen und internationalen Recht.

Anders als Erichsen und Seibt setzt Leonhards Definition des ökologischen Schadens im 1. Abschnitt des Buches (S. 24-46) nicht bei der individuellen Zuordnung der Umweltgüter an. Ein ökologischer Schaden ist jede erhebliche und nachhaltige Beeinträchtigung der Umweltmedien Boden, Wasser, Luft, der Tier- und Pflanzenwelt einschließlich der Mikroorganismen, des Klimas sowie des Wirkungsgefüges zwischen ihnen (S. $36 \mathrm{ff}$., 40 und 394). Es besteht zwar ein Grundkonsens, welche faktischen Phänomene gemeint sind; die

Sven Erichsen, Der ökologische Schaden im internationalen Umwelthaftungsrecht - Völkerrecht und Rechtsvergleichung, Frankfurt/M. 1993.

Christoph H. Seibt, Zivilrechtlicher Ausgleich ökologischer Schäden, Tübingen 1994. 